\title{
Automatic Localization and Boundary Detection of Optic Disc Using Implicit Active Contours
}

\author{
Siddalingaswamy P. C. \\ Department of Computer science \& Engineering \\ Manipal Institute of Technology \\ Manipal University, Manipal, India
}

\author{
Gopalakrishna Prabhu .K \\ Department of Biomedical Engineering \\ Manipal Institute of Technology \\ Manipal University, Manipal, India
}

\begin{abstract}
An efficient detection of Optic disc in colour retinal images is the fundamental step in an automated retinal image analysis system. This paper presents a new approach for the automatic localization and accurate boundary detection of the optic disc. Iterative thresholding method followed by connected component analysis is employed to locate the approximate center of the optic disc. Then geometric model based implicit active contour model is applied to find the exact boundary of the optic disc. The method is evaluated against a carefully selected database of 148 retinal images and compared with the human expert. The optic disc is localized with an accuracy of $99.3 \%$. The sensitivity and specificity of boundary detection achieved in terms of Mean \pm SD are $90.67 \pm 5$ and $94.06 \pm 5$ respectively.
\end{abstract}

\section{Keywords}

Optic disc segmentation, Iterative thresholding, Geometric active contours.

\section{INTRODUCTION}

Digital photography of the retina is widely used for screening of patients suffering from sight threatening diseases such as Diabetic retinopathy and Glaucoma. The timely diagnosis and referral for management of these diseases can prevent $98 \%$ of severe visual loss [1]. The shortage of the trained personnel in many countries leads to the need for automatic retinal image analysis system. Reliable and efficient automatic detection of normal features like optic disc, blood vessels and fovea in the retinal images are significant tasks in an automatic screening system. Optic disc detection is required as a prerequisite for the subsequent stages in many methods applied for identification of the pathological structures in retinal images. For example, in blood vessel tracking approaches the position of vessels in the neighbourhood of optic disc is used as seeds for vessel tracking [2]. In macula localization the approximate distance between optic disc and the macula is used as a priori knowledge for locating the macula [12]. In case of diabetic retinopathy lesions identification removing the false positive optic disc region leads to improved lesion diagnosis performance [11] [15]. The measurement of varying disc diameter is used in the detection of Glaucoma [8].

In the literature a very few work have been reported on locating the optic disc boundary in retinal images without user intervention. Most of the methods only locate the optic disc centre and do not address the problem of optic disc boundary localization. An edge detection stage followed by a circular Hough transform to locate the optic disc in the red colour component of the retinal image was reported in [6]. This approach rely on Sobel edge detection algorithm often fail to provide an acceptable solution due to the fuzzy boundaries and missing edge features. A method for locating the optic disc using a combination of pixel clustering and principal component analysis technique was reported in [3]. The technique was tested against 40 images and the results were reported satisfactory. In [13] a sub-image of specific size was used to evaluate the intensity variance of adjacent pixels, and marking the point with the largest variance as the optic disc location. However, the authors mainly focused on locating the optic disc centre and did not address the optic disc boundary localization. In [2] optic disc tracking was done by pyramidal decomposition technique followed by template matching using Hausdorff distance. But the technique needs prior information about the location of disc in the retinal image. The optic disc was located using fuzzy convergence of blood vessels in [4]. Here the implementation is difficult as the blood vessel tracking itself is a complicated task. In [15] the optic disc was located by means of mathematical morphology filtering techniques and watershed transformation. It is reported that this method was tested against a database of 30 retinal images out of that in 27 the exact contours were found. Parametric active contour model was used to detect the boundary of optic disc in [9] and [10]. Colour morphology in lab colour space followed by the contour detection snakes based on an external image field called Gradient Vector Flow was reported in [9].

In the proposed work the boundary of optic disc is located in two steps approach. First, the approximate center of optic disc is detected in the retinal image using iterative thresholding method followed by connected component analysis. It provides baseline for finding of its exact boundaries. Then, the geometric model based implicit active contour is employed to obtain accurate optic disc boundary. Geometric models differ from parametric models [9][10] in the sense that they do not depend much on image gradient and less sensitive to location of initial contour, thus performs better for object with week boundaries as in the case of optic disc. 


\section{MATERIALS AND METHOD}

Digital colour retinal images required for the automated optic disc segmentation were provided by of Department of Ophthalmology, Kasturba Medical College, Manipal. The images are captured using a Sony FF450IR digital fundus camera. A total of 148 images with resolution of $768 \times 576$ in 24bit JPEG format are used. The optic disc boundary in each of the image is hand labeled by an expert to be used as ground truth. The images are of very large variability in terms of lesions and image quality.

\subsection{Optic Disc Localization}

The optic disc is brighter than all other features in the retinal image, and it is observed that it appears most contrasted in the green channel compared to red and blue channels in RGB image. Therefore, only the green channel image is used for the effective thresholding of the optic disc. Optimal thresholding method [14] based on approximation of the histogram of an image using a weighted sum of two or more probability densities with normal distribution is applied to segment the brightest regions in the image. To obtain an optimal threshold, histogram derived from the source image is scanned from highest intensity $t_{1}$ value to lower intensity value. The scanning stops at the intensity level $t_{2}$ which has at least a thousand pixels with the same intensity resulting in a subset of histogram. From this the optimal threshold is calculated as follows.

1. Initial estimate of $\mathrm{T}$ is calculated at step $k$ as

$$
T_{k}=\frac{t_{1}+t_{2}}{2}
$$

2. Mean object gray level and background gray level is computed as follows

$$
\begin{aligned}
\mu_{b}^{k} & =\frac{\sum_{(i, j) \in \text { background }} I(i, j)}{\# \text { background pixels }} \text { and } \\
\mu_{o}^{k} & =\frac{\sum_{(i, j) \in \text { object }} I(i, j)}{\text { \# object pixels }}
\end{aligned}
$$

3. New threshold is calculated as

$$
T_{k+1}=\frac{\mu_{b}^{k}+\mu_{o}^{k}}{2}
$$

4. Step 2 to 3 is repeated until $T_{k+1}=T_{k}$

Optimal threshold thus calculated results in maximization of gray level variance between object and background. When applied to the image it results in number of isolated connected regions. Each of the connected components in the thresholded image is labeled, total number of pixels in the component and mean spatial co-ordinates of each is calculated. The component having the maximum number of pixels is assumed to be having the optic cup part of the optic disc and it is considered to be the primary region of interest. In the image the maximum diameter of optic disc can be 100 pixels. If any of the components whose mean spatial coordinates are within 50 to 60 pixels distance from the mean spatial coordinates of the largest component, then they are merged with it and new mean co-ordinate is calculated. If this region is greater than $80 \times 80$ pixels a rectangle is drawn to indicate the detection of approximate center of optic disc as shown in the Figure 1. Otherwise the threshold is decremented by one and applied to the initial image only in the local rectangular region around the candidate region. This iterative process is repeated until an optimal size of the optic disc has been obtained.

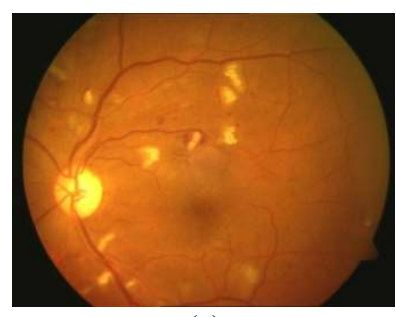

(a)

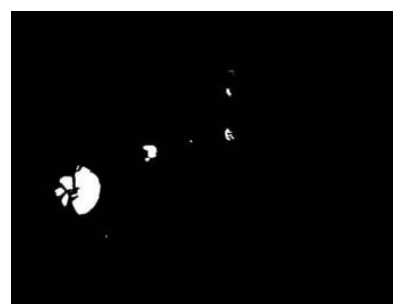

(c)

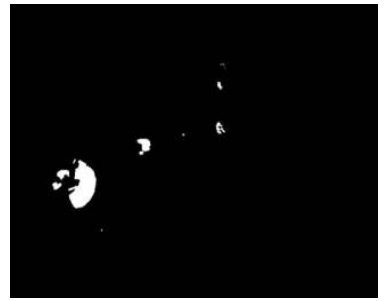

(b)

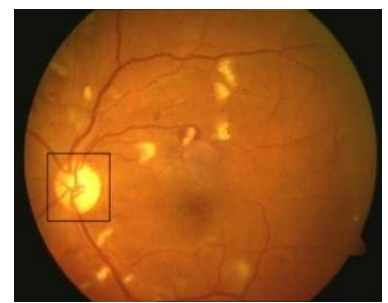

(d)
Figure 1. Optic Disc localization. (a) Input image. (b) Candidate regions after initial optimal thresholding. (c) Final thresholded image. (d) Localized optic disc.

\subsection{Optic Disc Boundary Detection}

The detection of accurate boundary of the optic disc is important for the detection and diagnosis of Glaucoma where the variation in the shape and size of the optic disk is used to detect and measure the severity of disease. Difficulty in finding the optic disc boundary is due to its highly variable appearance in retinal images. Classical segmentation algorithms such as edge detection, thresholding, and region grow are not enough to accurately find boundary of the optic disc as they do not incorporate the edge smoothness and continuity properties. In contrast, active contours (snakes) represent the paradigm that the presence of an edge depends not only on the gradient at a specific point but also on the spatial distribution [5]. Snakes are energy minimizing splines and are generally classified as parametric or geometric according to their representation. In the proposed work, the automatic optic disc boundary is detected by fitting an implicit active contour based on geometric model as reported in [7].

The optic disc region is usually fragmented into multiple subregions by blood vessels that have comparable gradient values. Therefore it cannot be directly segmented using snake algorithm. Use of median filter with appropriate size to remove interfering blood vessels from the optic disc region resulted in heavy blurring of disc boundaries. Instead a better result is achieved with gray level mathematical morphology to removes irrelevant vessels from the optic disc region. The closing of an image is defined as dilation followed by erosion and it tends to 
smooth the small-scale dark structures in an image. Denoting an image by $I$ and a structuring element by $S$, the dilation $\oplus$ and erosion $\Theta$ at a particular pixel $(x, y)$ is defined as:

$$
\begin{aligned}
& (I \oplus S)(x, y)=\max _{j, k}[I(x-j, y-k)+S(j, k)] \\
& (I \theta S)(x, y)=\min _{j, k}[I(x+j, y+k)-S(j, k)]
\end{aligned}
$$

As closing only eliminate image details smaller than the structuring element used, it is convenient to set the structuring element big enough to cover all possible vascular structures, but still small enough to keep the actual edge of the optic disc. Here, we used a $15 \times 15$ symmetrical disc structuring element, since the blood vessels are assumed to be not wider than 15 pixels in our colour retinal images. Figure 2 illustrates the gray scale morphology closing result on typical gray level retinal image. Dilation operation is done remove the blood vessels and then an erosion is done to restore the boundaries to their former position.

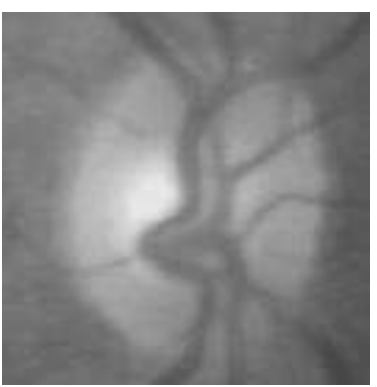

(a)

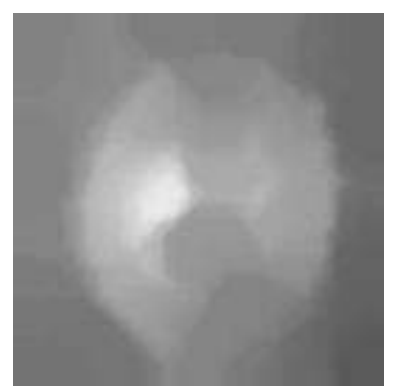

(b)
Figure 2. (a) Optic disc region fragmented by vessels. (b) Gray level morphologically closed optic disc region.

In geometric deformation model the curves are evolved implicitly using geometric computations. They perform image segmentation by starting with initial curve and evolve its shape by minimizing energy function represented by level set function $\varnothing$ in the image domain $\Omega$. The curve evolution stops at the image boundaries where the energy is minimum. In level set methods, a contour is represented by zero level set function $\varnothing$ and the energy function that is to be iteratively minimized to find the object boundary is given as follows.

$$
F_{\phi, f_{1}, f_{2}}=E_{\phi, f_{1}, f_{2}}+\mu P(\phi)+v L(\phi)
$$

Where $E_{\phi, f_{1}, f_{2}}$ is the external energy function, $\varnothing$ is the zero level set representing contour $C$ in the image domain, and are two values that fit the image intensities inside and outside the contour. $P(\varnothing)$ is the distance regularizing term used to penalize the deviation of level set $\varnothing$ from a signed distance function. It is given by

$$
P(\phi)=\int_{\Omega} \frac{1}{2}(|\nabla \phi(x)|-1)^{2} d x
$$

$L(\varnothing)$ is the length of zero level curve of $\varnothing$ used to regularize the contour. It is given by

$$
L(\phi)=\int_{\Omega} \delta(\phi(x))|\nabla \phi| d x
$$

$\mu$ and $v$ are positive constants, $\delta$ is the smoothing function called dirac function.

The energy functional (3) is to be minimized to find the optic disc boundary. Here gradient descent method is used to minimize the energy function and it is given by

$$
\begin{array}{r}
\frac{\partial \phi}{\partial t}=-\delta(\phi)\left(\alpha_{1} e_{1}-\alpha_{2} e_{2}\right)+v \delta(\phi) d i v\left(\frac{\nabla \phi}{|\nabla \phi|}\right) \\
+\mu\left(\nabla^{2} \phi-\operatorname{div} \frac{\nabla \phi}{|\nabla \phi|}\right)
\end{array}
$$

The functions $e_{1}$ and $e_{2}$ are calculated as follows

$$
\begin{aligned}
& e_{1}=\int_{\Omega} k_{\sigma}(y-x)\left|I(x)-f_{1}(y)\right|^{2} d y \\
& e_{2}=\int_{\Omega} k_{\sigma}(y-x)\left|I(x)-f_{2}(y)\right|^{2} d y
\end{aligned}
$$

where $\alpha_{1}$ and $\alpha_{2}$ are positive constants, $k_{\sigma}$ is the Gaussian kernel with localization property with $\sigma$ as scaling parameter.

The first term in (6) is called data fitting term responsible for driving the active contour toward object boundary. Second term is called length term and it has smoothing effect on contour. Third term is the level set regularization term that controls the speed of contour.

Once the vascular structures are removed based on the morphological pre-processing step, the boundary detection operation is carried out. To fit active contour onto the optic disc the initial contour must be near to the desired boundary otherwise it can converge to the wrong resting place. In order to automatically position an initial contour, the approximate center of optic disc obtained in the localization method is used. Figure 3(a) show the hand labeled optic disc boundary by an expert. The automatically detect optic disc boundary is overlapped on the ground truth image in Figure 3(b). Final result of the method is depicted shown in the Figure 3(c).

\section{RESULTS}

The optic disc is located correctly in 147 images out of 148 even in the presence of lesions in the images. In one image it was location with slight variation from actual location. Still it was considered for next step. In the detection of optic disc boundary using active contour model, we initialized the snake as a circle with optic disc centre obtained in localization method. Initial set of points that define contour of the snake are automatically selected in the $150 \times 150$ pixels region containing optic disc. Several different values were tested for the parameters of the gradient descent flow equation and we found the weights $\alpha_{1}=0.5, \alpha_{2}=0.5$ that are integrals over the region outside and inside contour as the best for our images. Length shortening term is set to $v=66$. Scaling parameter is set to $\sigma=2.0$ and $\mu=1$ as the regularization value. The number of iterations for convergence is set to 200 . With these parameter settings the snake algorithm is applied to 148 images in the dataset. The Hand labeled optic disc boundary for all the images is used as groundtruth. 


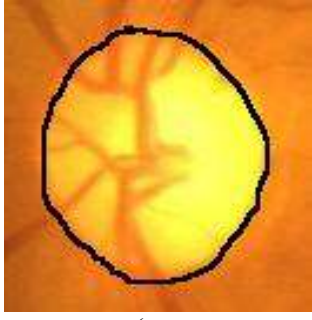

(a

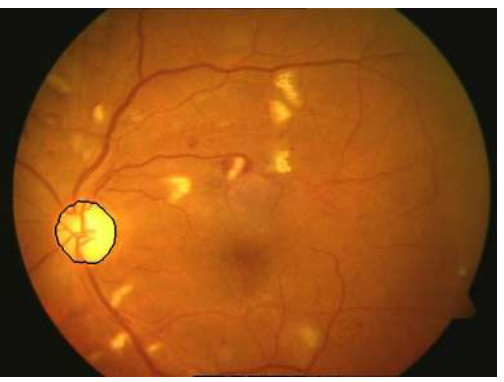

(c

Figure 3. (a) Hand labeled optic disc boundary. (b) Detected boundary overlaid on (a). (c) Final output

We quantify and compare the accuracy of the boundary localization against the hand labeled groundtruth produced by a clinician. Eq. (9) is used to calculate sensitivity and specificity which is used as the measure to match between two regions in the images.

$$
S_{n}=\frac{T_{p}}{T_{p}+F_{n}} \quad \text { and } \quad S_{p}=\frac{T_{n}}{T_{n}+F_{p}}
$$

where $T_{p}, T_{n}, F_{p}$ and $F_{n}$ are true positives, true negatives, false positives, and false negatives, respectively. Table 1 shows overall sensitivity and specificity in terms of mean and standard deviation for all 148 images in the dataset.

Table 1. Performance of optic disc boundary detection

\begin{tabular}{|c|c|c|}
\hline No. of images & Sensitivity & Specificity \\
\hline 148 & $90.67 \pm 5.05$ & $94.06 \pm 6.83$ \\
\hline
\end{tabular}

\section{CONCLUSION}

An efficient method for the automatic segmentation of optic disc boundary in colour retinal images is presented in this paper. Retinal images of patients at different stages of retinopathy were considered to test the robustness of the optimal iterative threshold method followed by connected component analysis and in most of the cases the optic disc was located correctly. Based on the result obtained in optic disc boundary detection, it can be stated that geometric based implicit active contour models provide a better segmentation for images with weak boundaries when compared to parametric models. It is hoped that the automatic optic disc segmentation method can assist the ophthalmologist for the early detection of glaucoma and retinopathy diseases.

\section{ACKNOWLEDGMENTS}

Our sincere thanks to ophthalmologists of the Department of Ophthalmology, Kasturba Medical College, Manipal for providing the necessary images and clinical details.

\section{REFERENCES}

[1] Emily, Y. 2003. Diabetic Retinopathy, American academy of ophthalmology - Retina panel, Preferred practice patterns.

[2] Gagnon, L., Lalonde, M., Beaulieu, M. 2001. Procedure to detect anatomical structures in optical fundus images. Proceedings of Conference Medical Imaging. (San Diego). 218-1225.

[3] Huiqi Li and Opas Chutatape. 2004. Automated Feature Extraction in Color Retinal Images by a Model Based Approach. IEEE Trans. Biomedical Engineering. vol. 51, no. 2, 246-254.

[4] Hoover, A. and Goldbaum, M. 2003. Locating the Optic Nerve in a Retinal Image Using the Fuzzy Convergence of the Blood Vessels. IEEE Trans. Med. Imaging. vol. 22, no. $8,951-958$.

[5] Kass, M., Witkin, A. and D. Terzopoulos. 1987. Snakes: active contour models. Int. J. Comput. Vis. vol. 1, pp. 321331

[6] Liu, Z., Chutatape, O. and Krishnan, S. M. 1997. Automatic image analysis of fundus photograph. Proceedings of 19th Annual Int. Conf. IEEE Engineering in Med. and Biology Society. vol. 2, 524-525.

[7] Li, C., Kao, C., Gore, J. and Ding, Z. 2007. Implicit active contours driven by local binary fitting energy. IEEE Conf. Computer Vision and Pattern Recognition.

[8] Marios, C., Ferraro J., Ecosse L., Taylor, H. 2005. Assessment of Optic Disc Cupping With Digital Fundus Photographs. American Journal of Ophthalmology. vol. 140 , no. 3, 529-531.

[9] Mendels, F., Heneghan, C. and Thiran, J. P. 1999. Identification of the Optic Disk Boundary in Retinal Images Using Active Contours. Proc. IMVIP, 103-115.

[10] Osareh, A., Mirmehdi, M., Thomas, B and Markham, R. Colour Morphology and Snakes for Optic Disc Localisation. 2002. Proc. $6^{\text {th }}$ Medical Image Understanding and Analysis Conference. 21-24.

[11] Osareh, A., Mirmehdi, M., Thomas, B and Markham, R. 2007. Automated identification of diabetic retinal exudates in digital colour images. British Journal of Ophthalmology. vol. 87, 1220-1223, 2007.

[12] Siddalingaswamy, P. C. and Prabhu, G. K. 2007. Automated Detection of Anatomical Structures in Retinal Images. $7^{\text {th }}$ IEEE International Conference on Computational Intelligence and Multimedia Applications. vol. $3,164-168$. 
[13] Sinthanayothin, C., Boyce, J. F., Cook, H. L. and Williamson, T. H. 1999. Automated location of the optic disc, fovea, and retinal blood vessels from digital color fundus images. British Journal of Ophthalmology. vol. 83, no. 8, pp. 902-910.

[14] Sonka M, Hlavac V and Boyle R 2008 Digital image processing and Computer vision Cengage Learning India pvt. Ltd. 157-160.
[15] Walter, T., Klein, J. C., Massin, P. and Erginay, A. 2002. A contribution of Image Processing to the Diagnosis of Diabetic Retinopathy-Detection of Exudates in Color Fundus Images of the Human Retina. IEEE Trans. Medical. Imaging, vol. 21, no. 10. 1236-1243. 